\title{
Fatores associados à polifarmácia em idosos institucionalizados
}

Factors associated to polypharmacy in institutionalized elderly

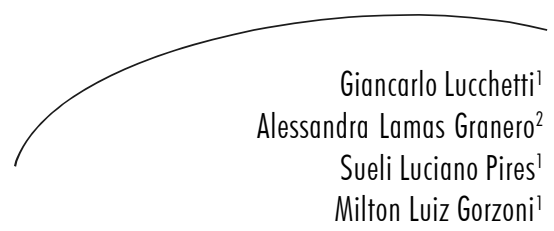

Resumo

Introdução: A polifarmácia é um problema importante no atendimento do idoso, sendo os pacientes em instituições de longa permanência (ILPs) aqueles com riscos aumentados, por apresentarem muitas doenças limitantes, fragilidade e baixa funcionalidade. Objetiva-se então, com o presente estudo, avaliar os fatores associados à presença de polifarmácia em pacientes idosos internados em instituição de longa permanência. Metodologia: Realizado estudo transversal, retrospectivo, por meio de análise de prontuários de todos os pacientes acima de 60 anos institucionalizados em ILP pública de grande porte. Elaborou-se protocolo constituído de dados sócio-demográficos, número e tipos de doenças, número de medicamentos, grupos de fármacos, dependência funcional e tempo de institucionalização. $\mathrm{Na}$ análise estatística, esses fatores foram relacionados com a presença ou não de polifarmácia. Resultados: A casuística final constituiu-se de 209 pacientes; 73,2\% mulheres, idade média de 75,9 anos e tempo de institucionalização médio de 95,3 meses. Principais doenças encontradas: hipertensão arterial sistêmica (HAS) em 67,9\% do total, síndromes demenciais em $36,8 \%$ e sequelas de trauma crânio-encefálico ou acidente vascular cerebral (TCE ou AVC) em 33,9\%. No presente estudo, os fatores relacionados à polifarmácia em institucionalizados foram: ausência de déficit cognitivo $(\mathrm{OR}=3,61$ - IC: $1,96-6,65)$; consumo de medicamentos cardiovasculares $(\mathrm{OR}=0,22$ - IC: 0,11-0,44), uso de medicamentos gastrointestinais e metabolismo $(\mathrm{OR}=0,38$ - IC: 0,21-0,67); número de diagnósticos acima de 5 (OR=1,90 - IC: 1,08-3,33); tempo de institucionalização entre 12 e 23 meses (OR:0,32 - IC: 0,11-0,91) e maior dependência funcional pela escala de Katz (OR: 1,90 - IC:1,07-3,22). Não houve relação com idade, sexo, uso de medicamentos psiquiátricos e diagnóstico de sequela de AVC ou TCE. Conclusão: Conclui-se que institucionalizados possuem aspectos diferentes de idosos ambulatoriais ou de comunidade, havendo maior importância de fatores relacionados à funcionalidade e ao tempo de institucionalização do que com idade e sexo.

Setor de Geriatria do Departamento de Medicina da Irmandade da Santa Casa de Misericórdia de São Paulo. São Paulo, SP, Brasil

2 Hospital Geriátrico e de Convalescentes Dom Pedro II (HGCDP). São Paulo, SP, Brasil

Correspondência / Correspondence

Giancarlo Lucchetti

E-mail: gian.tln@terra.com.br

Palavras-chave: Saúde do Idoso Institucionalizado. Avaliação de Medicamentos. Instituição de Longa Permanência para Idosos ; Doença Iatrogênica; Estudos Transversais. Polifarmácia. Iatrogenia. 


\section{Abstract}

Introduction: Polypharmacy is an important issue in the elderly care. The ones under institutionalization have higher risks, due to their comorbidities, frailty and dependency. This study aims to evaluate the factors associated with polypharmacy in institutionalized elderly. Methods: A retrospective, crosssectional study was carried out. All medical charts of patients aged 60 years old or more in a huge public nursing home were evaluated. A protocol containing socio-demographic data, number and types of diseases, number of drugs, types of drugs, functional status and time of institutionalization were analyzed. Through statistical analysis, these factors were linked or not to polypharmacy.Results: The final sample comprised 209 patients, $73.2 \%$ women, average age of 75.9 years and with an institutionalized time of 95.3 months. The most prevalent diseases were: arterial hypertension $-67.9 \%$, dementia $-36.8 \%$ and stroke or head trauma sequelae $-33.9 \%$. In this study, the associated factors related to polypharmacy in nursing home residents were: lack of cognitive deficit, $(\mathrm{OR}=3,61$ - IC: 1,96-6,65), use of cardiovascular drugs ( $\mathrm{OR}=0,22-\mathrm{IC}: 0,11-0,44)$, use of gastrointestinal and metabolic drugs $(\mathrm{OR}=0,38$ - IC: 0,21-0,67), five or more diagnosis $(\mathrm{OR}=1,90-\mathrm{IC}$ : 1,08-3,33), institutionalized time between 12 and 23 months (OR:0,32 - IC: 0,11$0,91)$ and greater dependency in Katz scale (OR: 1,90 - IC:1,07-3,22). There were no relationship with age, sex, psychiatric drugs and diagnosis of stroke or head trauma sequelae. Conclusion: It follows that institutionalized elderly have different aspects as compared to outpatients or community, with major factors related to functionality and time of institutionalization than to age and sex.
Key words: Health of Institutionalized Elderly. Drug Evaluation. Homes for the Aged. Iatrogenic Disease. Cross-Sectional Studies. Polypharmacy. Nursing homes. Elderly. Iatrogeny.

\section{INTRODUÇÃO}

A polifarmácia é um problema importante no atendimento do idoso, devendo ser sempre pesquisada e evitada quando possível. O paciente geronte é mais propenso ao uso de muitos medicamentos devido ao fato de ter mais comorbidades, das alterações fisiológicas de farmacocinética e farmacodinâmica ${ }^{1}$ e da própria idade. $^{2}$

Onúmero de medicamentoséo principal fator de risco para iatrogenia e reações adversas, havendo relação exponencial entre a polifarmácia e a probabilidade de reação adversa, interações medicamentosas e medicamentosinapropriados para idosos. ${ }^{3}$

Alguns fatores têm sido correlacionados com a presença de polifarmácia com o intuito da identificação dos grupos mais susceptíveis a iatrogenia, como por exemplo, a idade, a funcionalidade e as doenças crônicas. ${ }^{4}$ Sendo assim, os pacientes em instituições de longa permanência
(ILPs) seriam aqueles com riscos aumentados, por apresentarem muitas doenças limitantes, fragilidade e baixa funcionalidade. Essa parcela da população já representa nos países em transição demográfica avançada $11 \%, 5$ enquanto que no Brasil chega a $1,5 \%,{ }^{6}$ sendo que a tendência, na medida em que nosso próprio país envelhece, é que a demanda para tais serviços cresça ainda mais.

Muitos estudos têm pesquisado o uso de medicamentos e a presença de polifarmácia em pacientes idosos ambulatoriais, em inquéritos populacionais e em pacientes hospitalizados. Entretanto, ainda são escassos em nosso meio estudos que demonstrem a relação entre polifarmácia e fatores de risco em institucionalizados, de forma que se possa pensar nos fatores de risco e fomentar meios para uma intervenção antes de ser instalada a polifarmácia. Objetiva-se, com o presente estudo, avaliar quais são os fatores relacionados à presença de polifarmácia ou não em pacientes idosos internados em Instituição de Longa Permanência. 


\section{CASUÍSTICA E MÉTODOS}

Realizou-se estudo transversal, retrospectivo, por meio de análise de prontuários de todos os pacientes acima de 60 anos do Hospital Geriátrico e de Convalescentes Dom Pedro II, da Irmandade da Santa Casa de Misericórdia de São Paulo, no mês de fevereiro de 2005. Incluíram-se no estudo pacientes com 60 anos ou mais, independentemente do padrão de morbidade apresentada, sendo excluídos institucionalizados com idade menor de 60 anos, internados na unidade de eventos agudos, os que evoluíram para óbito durante o estudo e os com prontuários sem todos os dados do protocolo.

Elaborou-se protocolo constituído de dados sócio-demográficos (contendo unidade a que pertenciam, iniciais do nome, registro, sexo, idade e tempo de institucionalização), número e tipos de doenças (enumeradas conforme expostas no prontuário médico), número de medicamentos e presença de polifarmácia (definida no presente estudo como mais de cinco medicamentos por paciente), ${ }^{7}$ grupos de fármacos (divididos nos grupos cardiovasculares, gastrointestinais e metabolismo, e psiquiátricos), dependência funcional (pela escala de Katz ${ }^{8}$ dividindo-se de 0 a 6, conforme grau de independência, sendo: 2 ou menos - muito dependente; 3 e 4 - dependente parcial; e acima ou igual a 5 - independente) e tempo de institucionalização (quantos meses desde o ingresso na ILP). Os protocolos foram preenchidos por médicos após minuciosa revisão dos prontuários, seguindo-se os critérios de inclusão e exclusão.

Os dados foram tabulados pelo programa Excel for Windows. A análise estatística calculou medidas-resumo para variáveis quantitativas e, para as qualitativas, foram apresentadas frequências absolutas e relativas e construídas tabelas de contingência. Considerou-se como desfecho a variável polifarmácia e, para compará-la com outras variáveis qualitativas, utilizou-se a análise bivariada mediante o teste de associação de Quiquadrado. Calculou-se a razão de chances (Odds Ratio) com intervalo de confiabilidade de $95 \%$. $O$ valor de $\mathrm{p}<0,05$ foi considerado estatisticamente significante. As análises foram feitas pelo programa Epi info versão 3.5.

O presente estudo foi aprovado pelo Comitê de Ética em Pesquisa da Irmandade da Santa Casa de Misericórdia de São Paulo, mediante protocolo número 308/05.

\section{RESULTADOS}

Avaliaram-se todos os internados do Hospital Geriátrico e de Convalescentes Dom Pedro II (instituição de longa permanência para idosos vinculada à Irmandade da Santa Casa de Misericórdia de São Paulo e que disponibiliza vagas para 20 hospitais e 3 pronto-socorros de São Paulo), totalizando 505 leitos e pacientes. Destes, 197 foram excluídos por apresentarem menos de 60 anos de idade, 15 por estarem internados na unidade de agudos, 81 devido à falta de dados durante a coleta e três por óbito. A casuística final constituiu-se de 209 pacientes: 153 mulheres ( $73,2 \%$ do total) e 53 homens (26,8\%), com idade média de 75,9 $\pm 10,7$ anos e tempo de institucionalização de 95,3 +30 meses.

As principais doenças encontradas foram: (1) hipertensão arterial sistêmica (HAS) em 142 internados (67,9\% do total); (2) síndromes demenciais em 77 (36,8\%); (3) sequelas de trauma crânioencefálico ou acidente vascular cerebral (TCE ou AVC) em 71 (33,97\%); e (4) doenças cardiovasculares em 70 pacientes (33,4\%). Observou-se polifarmácia em 97 idosos (46,4\% do total), e o principal grupo de fármacos usado foi o de medicamentos cardiovasculares, seguidos dos psicotrópicos (neurolépticos, benzodiazepínicos, anticonvulsivantes, antidepressivos), conforme a tabela 1 . 
Tabela1-Principais grupos de medicações utilizados pelos idosos internados no Hospital Geriátrico e de Convalescentes Dom Pedro II. São Paulo, SP, Brasil, 2005.

\begin{tabular}{lcc}
\hline \multicolumn{1}{c}{ Medicamentos } & No de idosos & Frequência \\
\hline Cardiovasculares & 147 & $70,3 \%$ \\
Psicotrópicos & 105 & $50,3 \%$ \\
Gastrointestinais e & 103 & $49,3 \%$ \\
Metabolismo & 50 & $23,9 \%$ \\
Vitaminas & & \\
\hline
\end{tabular}

Foi realizada análise estatística tendo como variável dependente a polifarmácia e correlacionando com variáveis independentes (sexo, presença de demência, presença de sequela de AVC/TCE, uso de medicamentos cardiovasculares, gastrointestinais e psiquiátricos, idade, tempo de institucionalização, número de diagnósticos e grau de funcionalidade pela escala de Katz), obtendo-se valores de significância para $\mathrm{p}<0,05$ (tabela 2).

Tabela 2 - Análise bivariada da associação entre polifarmácia e dados demográficos, comorbidades, tipos de medicamentos, tempo de institucionalização e funcionalidade, em institucionalizados no Hospital Geriátrico e de Convalescentes Dom Pedro II. São Paulo, SP, Brasil, 2005.

\begin{tabular}{|c|c|c|c|c|}
\hline Variável & $\begin{array}{c}\text { Não } \\
\text { Polifarmácia }\end{array}$ & Polifarmácia & Significância* & OR (IC 95\%) \\
\hline \multicolumn{5}{|l|}{ Sexo: } \\
\hline Masculino ${ }^{1}$ & $35(31,3 \%)$ & $21(21,6 \%)$ & \multirow[t]{2}{*}{$p=0,110$} & 1,00 \\
\hline Feminino & $77(68,8 \%)$ & $76(78,4 \%)$ & & $1,64(0,87-3,07)$ \\
\hline \multicolumn{5}{|l|}{ Demência: } \\
\hline $\operatorname{Sim}^{1}$ & $56(50 \%)$ & $21(21,6 \%)$ & \multirow[t]{2}{*}{$\mathrm{P}<0,05^{* *}$} & 1,00 \\
\hline Não & $56(50 \%)$ & $76(78,4 \%)$ & & $3,61(1,96-6,65)$ \\
\hline \multicolumn{5}{|l|}{ Sequela AVC/TCE: } \\
\hline $\operatorname{Sim}^{1}$ & $37(33 \%)$ & $34(35,1 \%)$ & \multirow[b]{2}{*}{$\mathrm{p}=0,75$} & 1,00 \\
\hline Não & $75(67 \%)$ & $63(64,9 \%)$ & & $0,91(0,51-1,62)$ \\
\hline \multicolumn{5}{|l|}{ Medicamento Cardiovascular } \\
\hline $\operatorname{Sim}^{1}$ & $64(57,1 \%)$ & $83(85,6 \%)$ & \multirow[t]{2}{*}{$\mathrm{P}<0,05^{* *}$} & 1,00 \\
\hline Não & $48(42,9 \%)$ & $14(14,4 \%)$ & & $0,22(0,11-0,44)$ \\
\hline \multirow{3}{*}{\multicolumn{5}{|c|}{$\begin{array}{l}\text { Medicamento } \\
\text { Gastrointestinal e } \\
\text { Metabolismo }\end{array}$}} \\
\hline & & & & \\
\hline & & & & \\
\hline $\operatorname{Sim}^{1}$ & $43(38,4 \%)$ & $60(61,9 \%)$ & \multirow[t]{2}{*}{$\mathrm{P}<0,05 \%$} & 1,00 \\
\hline Não & $69(61,6 \%)$ & $37(38,1 \%)$ & & $0,38(0,21-0,67)$ \\
\hline \multicolumn{5}{|l|}{ Medicamento psiquiátrico } \\
\hline $\operatorname{Sim}^{1}$ & $59(52,7 \%)$ & $52(53,6 \%)$ & \multirow{2}{*}{$\mathrm{p}=0,89$} & 1,00 \\
\hline Não & $53(47,3 \%)$ & $45(46,4 \%)$ & & $0,96(0,55-1,66)$ \\
\hline \multicolumn{5}{|l|}{ Idade (anos) } \\
\hline 60 a 79 anos & $68(60,7 \%)$ & $62(63,9 \%)$ & \multirow[t]{2}{*}{$\mathrm{p}=0,31$} & $0,87(0,49-1,53)$ \\
\hline Acima de 80 anos $^{1}$ & $44(39,3 \%)$ & $35(36,2 \%)$ & & 1,00 \\
\hline \multicolumn{5}{|l|}{$\begin{array}{l}\text { Tempo de institucionalização } \\
\text { (meses) }\end{array}$} \\
\hline O a 11 meses $^{1}$ & $22(20,2 \%)$ & $25(26,6 \%)$ & \multirow{4}{*}{$\mathrm{P}<0,05 \%$} & 1,00 \\
\hline 12 a 23 meses & $19(17,4 \%)$ & $7(7,4 \%)$ & & $0,32(0,11-0,91)$ \\
\hline \multirow{2}{*}{ Acima de 36 meses } & $13(11,9 \%)$ & $4(4,3 \%)$ & & $0,27(0,07-0,95)$ \\
\hline & $55(50,5 \%)$ & $58(61,7 \%)$ & & $0,92(0,46-1,83)$ \\
\hline Número de diagnósticos & & & & 1,00 \\
\hline O a 4 diagnósticos & $75(67 \%)$ & $50(51,5 \%)$ & & 1,00 \\
\hline 5 ou mais diagnósticos & $37(33 \%)$ & $47(48,5 \%)$ & $\mathrm{P}<0,05^{* *}$ & $1,90(1,08-3,33)$ \\
\hline Escore de Katz & $68(60,7 \%)$ & $44(45,4 \%)$ & & 1,00 \\
\hline $\begin{array}{l}\text { Independente } \\
\text { Dependente Parcial e Total }\end{array}$ & $44(39,3 \%)$ & $53(54,6 \%)$ & $\mathrm{p}<0,05^{* *}$ & $1,86(1,07-3,22)$ \\
\hline
\end{tabular}

* Utilizado o teste de Qui-quadrado

** nível de significância para $\mathrm{p}<0,05$

AVC - acidente vascular cerebral

TCE - trauma crânio-encefálico

${ }^{1}$ classe adotada como referência na interpretação do OR (Odds Ratio ou Razão de Chance) 


\section{DISCUSSÃO E CONCLUSÃO}

Polifarmácia em idosos tornou-se significante aspecto na assistência geriátrica. Segundo Isaacs, ${ }^{9}$ a iatrogenia é um dos gigantes da geriatria e deve ser sempre investigada na consulta do paciente idoso. A prevalência de polifarmácia (definida como a tomada de mais de 5 medicamentos por paciente) $)^{7}$ é alta em diversos setores de atendimento e de atenção à saúde. Estudos observaram polifarmácia em $27,0 \%$ dos pacientes ambulatoriais residentes na Região Sul:;0 14,3\% em Belo Horizonte ${ }^{11}$ e 31,0\% no município de São Paulo. ${ }^{12}$ Foram encontradas também médias de medicamentos tomados por paciente de 9,9 a 13,6 em hospitalizados ${ }^{13}$ e de até sete em Unidade de Terapia Intensiva. ${ }^{14}$ Institucionalizados apresentaram consumo médio de 7,2 a 8,1 medicamentos por paciente ${ }^{15}$ nos Estados Unidos, e entre 4,7 e 4,6 no Brasil, ${ }^{16,17}$ com prevalência de polifarmácia em $47,7 \%$ dos pacientes, compatível com o presente estudo. Entretanto, alguns estudos recentes, como o conduzido por Hanlon et al. em 2009 , demonstraram até $74 \%$ de polifarmácia. ${ }^{18}$ Segundo outro estudo, conduzido na Finlândia, ${ }^{19}$ cada institucionalizado consumia em média 7,9 drogas, demonstrando que o problema da polifarmácia em ILPs se faz ainda atual.

Alguns autores consideram polifarmácia como a tomada simultânea de dois a quatro medicamentos (polifarmácia menor) e cinco ou mais (polifarmácia maior).$^{20}$ Nesta outra definição, a casuística estudada apresentaria polifarmácia em $93,8 \%$ dos idosos, sendo $47,4 \%$ com polifarmácia menor e $46,4 \%$ com polifarmácia maior.

Considerando-se o presente e universal consumo medicamentoso, particularmente alto em idosos, devem-se identificar fatores associados à polifarmácia em pacientes desta faixa etária. Casuísticas ambulatoriais notam possíveis associações da polifarmácia com sexo feminino, ${ }^{4,10,11,21}$ idade avançada, ${ }^{4,11,19}$ maior número de consultas no último ano, 4,10,11,21 maior renda familiar, ${ }^{4,21}$ número de comorbidades, ${ }^{4,11}$ morar sozinho ${ }^{10}$ e ausência de déficit cognitivo (demência). ${ }^{22}$ Há poucos estudos na literatura consultada que avaliaram idosos institucio- nalizados, merecedores de atenção pela crescente expansão devido ao aumento de longevidade, morbidade e problemas sociais. Polifarmácia possui associação com reações adversas aos medicamentos, quedas, ${ }^{23,24}$ desnutrição,${ }^{25}$ prescrição de medicamentos inapropriados ${ }^{26} \mathrm{e}$ qualidade do sono. ${ }^{27}$ Entretanto não foram encontrados, na literatura consultada, estudos em institucionalizados, cujo desfecho final seja a própria polifarmácia.

No presente estudo, fatores relacionados à polifarmácia em institucionalizados foram: (1) ausência de déficit cognitivo (demência); (2) consumo de medicamentos cardiovasculares; (3) uso de medicamentos gastrointestinais e metabolismo; (4) número de diagnósticos acima de 5; (5) tempo de institucionalização; e (6) maior dependência funcional pela escala de Katz. Não houve relação com idade, sexo, uso de medicamentos psiquiátricos e diagnóstico de sequela de AVC ou TCE.

Correlacionam-se faixa etária e polifarmácia em vários trabalhos, idosos da comunidade apresentam maior consumo medicamentoso quanto mais avançada a idade. ${ }^{4,11,21}$ No entanto, neste estudo não houve esta diferença. Justificase pelo fato de a casuística incluir apenas idosos e uma eventual uniformidade de estado funcional por serem institucionalizados.

Não houve também relação entre polifarmácia e gênero, dado diferente dos estudos ambulatoriais e de comunidade. Esse dado pode ser justificado pelo próprio estado funcional dos residentes $\mathrm{da}$ ILP, conforme comentado acima.

Portadores de demência apresentam menor percentual de polifarmácia, ${ }^{22}$ dado compatível com o presente estudo em que $72,0 \%$ dos pacientes com síndrome demencial incluíam-se no grupo sem polifarmácia. Pressupõe-se que isto se deva à dificuldade de avaliação clínica de pacientes com disfunção cognitiva, gerando subutilização terapêutica.

O número de diagnósticos foi diretamente proporcional ao uso de medicamentos, concordando com a literatura consultada ${ }^{4,11} \mathrm{e}$ 
coerente com o senso comum de que quanto mais comorbidades maior seria o número de medicamentos necessários.

A funcionalidade foi inversamente proporcional à polifarmácia, melhor independência, menor uso de medicamentos, dado pouco abordado em estudos ambulatoriais, mas de profunda importância no contexto de pacientes em ILPs. Dependência maior associa-se habitualmente a maiores comorbidades, justificando assim o consumo de mais classes medicamentosas.

A significância de drogas cardiovasculares, gastrointestinais e metabolismo com a polifarmácia é justificada pelo fato de que pacientes que fazem uso desses medicamentos costumam utilizar associações medicamentosas, como no tratamento da hipertensão, arritmias, dislipidemia e síndrome metabólica.

Institucionalização obteve significância estatística de forma bimodal, ou seja, os institucionalizados recentes (até um ano) e aqueles com mais tempo de institucionalização (acima de 3 anos) possuíram mais polifarmácia. Esses dados correlacionam-se a que pacientes com institucionalização recente normalmente são egressos de internações hospitalares em circunstâncias de gravidade e instabilidade, o que propicia a prescrição de vários medicamentos simultaneamente. Já nos institucionalizados crônicos, declínio funcional e consumo medicamentoso são correlatos e progressivamente maiores.

Dos fatores associados e estatisticamente significantes, a maioria se enquadra na categoria dos não-modificáveis, como é o caso da presença de demência, o número de diagnósticos e o tempo de institucionalização. Entretanto, alerta-nos quanto à necessidade de uma revisão contínua das prescrições nesse tipo de pacientes e um maior cuidado na prescrição de novos fármacos, visando a uma menor instalação de polifarmácia, se possível. Quanto à funcionalidade, objetiva-se sempre um trabalho de reabilitação precoce pela ILP, no intuito de ter um menor declínio funcional e, consequentemente, uma diminuição na polifarmácia.

Recentemente, foi realizada uma meta-análise sobre a otimização dos medicamentos usados em ILPs por farmacêuticos. Segundo esse estudo, o farmacêutico teria papel importante no manejo e conhecimento dos medicamentos pelos médicos e enfermeiras, podendo ser uma estratégia para controlar a polifarmácia nesses locais ${ }^{28}$.

O estudo em questão possui algumas limitações que devem ser consideradas na interpretação dos resultados. Primeiro, trata-se de um estudo transversal, ou seja, apesar de encontrar fatores relacionados à polifarmácia, não demonstra uma relação de causa-efeito. Segundo, apesar de ser uma instituição de grande porte, é dirigida por uma instituição universitária e pode não representar a realidade de algumas ILPs. Terceiro, o perfil de dependência dos pacientes é alto e pode diferir de instituições de menores portes.

Conclui-se que, neste estudo, os institucionalizados possuem aspectos diferentes de idosos ambulatoriais ou de comunidade, havendo maior importância a fatores relacionados com funcionalidade e tempo de institucionalização do que com idade e sexo. Necessita-se, assim, de mais estudos que avaliem fatores relacionados à polifarmácia em pacientes em ILPs, para que se possam identificar fatores de risco e agir de forma a tentar evitar maior prescrição de medicamentos e seus efeitos adversos. 


\section{REFERÊNCIAS}

1. Carvalho Filho ET. Fisiologia do envelhecimento. In: Papaléo Netto M, organizador. Gerontologia: a velhice e o envelhecimento em visão globalizada. São Paulo: Atheneu; 1996. p. 60-70.

2. Rozenfeld S. Prevalência, fatores associados e mau uso de medicamentos entre os idosos: uma revisão. Rev Saude Publica 2003; 19(3):717-24.

3. Passarelli MCG, Gorzoni ML. Iatrogenia: Reações adversas a medicamentos. In: Jacob Filho W, Gorzoni ML. Geriatria e Gerontologia: o que todos deviam saber. São Paulo: Roca; 2008. p. 19-30.

4. Coelho Filho JM, Marcopito LF, Castelo A. Perfil de utilização de medicamentos por idosos em área urbana do Nordeste do Brasil. Rev Saude Publica 2004; 38 (4): 557- 64.

5. Chaimowicz F, Greco DB. Dinâmica da institucionalização de idosos em Belo Horizonte, Brasil. Rev Saude Publica [Online] 1999 out; 33(5) [8 Telas]. Disponível em: URL: http://www.fsp.usp.br/rsp.

6. Camarano AA. Brazilian population ageing: differences in well-being by rural and urban areas. IPEA. Texto Para Discussão [online] Maio 2002. [33 Telas] Disponível em: URL: http:// www.ipea.gov.br.

7. Carlson JE. Perils of polypharmacy: 10 steps to prudent prescribing. Geriatrics. 1996; 51(7):26-35.

8. Isaacs B. The giants of geriatrics: A study of symptons in old age. An inaugural lecture delivered in the University of Birminham, 1976, p. 1-13.

9. Lino VTS, Pereira SRM, Camacho LAB, et al. Adaptação transcultural da Escala de Independência em Atividades da Vida Diária (Escala de Katz). Cad Saude Publica 2008; 24(1): 103-12.

10. Flores LM, Mengue SS. Uso de medicamentos por idosos em região do sul do Brasil. Rev Saude Publica 2005; 39(6): 924-9.

11. Loyola Filho AI, Uchoa E, Lima-Costa MF. Estudo epidemiológico de base populacional sobre uso de medicamentos entre idosos na Região Metropolitana de Belo Horizonte, Minas Gerais, Brasil. Cad Saúde Pública 2006, 22(12):2657-67.

12. Carvalho MFC. A polifarmácia em idosos no município de São Paulo - Estudo SABE - Saúde, Bem-estar e Envelhecimento. [dissertação]. São Paulo: Universidade de São Paulo, Faculdade de Saúde Pública; 2007. 127p. tab.
13. Passarelli MC, Jacob-Filho W, Figueras A. Adverse drug reactions in an elderly hospitalised population: inappropriate prescription is a leading cause. Drugs Aging. 2005; 22(9):767-77.

14. N Buchanan, RD Cane. Drug utilization in a general intensive care unit. Intensive Care Med 1978; 4(2): 75-7.

15. Broderick E. Prescribing patterns for nursing home residents in the US. The reality and the vision. Drugs Aging 1997; 11(4):255-60.

16. Danilow MZ, Moreira ACS. Perfil epidemiológico, sociodemográfico e psicossocial de idosos institucionalizados do Distrito Federal. Comunicação em ciencias da saude 2007; 18(1):9-16.

17. Silva AEC, Menezes EAG, Coelho TOA, Moraes EM. Aspectos bio-psico-sociais dos idosos institucionalizados na Casa do Ancião da Cidade Ozanan, [acesso 2008 jul 19]. Anais do 8. Encontro de Extensão da UFMG. 2005, Belo Horizonte. Disponível em: http:// www.portaldoenvelhecimento.net/modos/ aspectos.pdf.

18. Hanlon JT, Wang X, Good CB, Rossi MI, Stone RA, Selma TP, Handler SM. Racial differences in medication use among older, long-stay Veterans Affairs nursing home care unit patients. Consult Pharm 2009 Jun;24(6):439-46.

19. Hosia-Randell HM, Muurinen SM, Pitkälä KH. Exposure to potentially inappropriate drugs and drug-drug interactions in elderly nursing home residents in Helsinki, Finland: a cross-sectional study. Drugs Aging. 2008;25(8):683-92.

20. Bjerrum L, Rosholm JU, Hallas J, Kragstrup J. Methods for estimating the occurrence of polypharmacy by means of a prescription database. Eur J Clin Pharmacol 1997; 53(1): 7-11.

21. Loyola Filho AI, Uchoa E, Firmo JOA, LimaCosta MF. Estudo de base populacional sobre o consumo de medicamentos entre idosos: Projeto Bambuí. Cad Saúde Pública 2005; 21(2): 545-53.

22. Loyola Filho AI, Uchoa E, Firmo JOA, LimaCosta MF. Influência da renda na associação entre disfunção cognitiva e polifarmácia: Projeto Bambuí. Cad Saude Publica 2008; 42(1): 89-99.

23. Field TS, Gurwitz MD, Avorn J. Risk factors for adverse drug events among nursing home residents. Arch Intern Med 2001; 161:1629-34.

24. Robbins AS, Rubenstein LZ, Josephson KR, Schulman BL, Osterweil D, Fine G. Predictors 
of falls among elderly people. Results of two population-based studies. Arch Intern Med 1989; 149:1628-33.

25. Amer, MS, Mousa SM, Abdel Rahman, TT, Saber, HG. Malnutrition and its risk factors in nursing home residents in Cairo. J Am Geriatr Soc 2009; 57(9):1716-8.

26. Ruths $S$. Evaluation of prescribing quality in nursing homes based on drug-specific indicators: The Bergen district nursing home
(BEDNURS) study. Nor Epidemiol 2008; 18 (2): 173-8.

27. Neikrug AB, Ancoli-Israel S. Sleep Disorders in the Older Adult: a mini-review. Gerontology 2009 [no prelo].

28. Verrue CL, Petrovic M, Mehuys E, Remon JP, Vander Stichele R. Pharmacists' interventions for optimization of medication use in nursing homes : a systematic review. Drugs Aging 2009;26(1):37-49.

Recebido: 07/4/2009

Revisado: 27/9/2009

Aprovado: 29/10/2009 